\title{
Potential of Standard Model measurements with CMS
}

\section{Jorgen D'Hondt* ${ }^{* \dagger}$}

Vrije Universiteit Brussel, Belgium

E-mail: jdhondtahep.iihe.ac.be

\begin{abstract}
Although hadron colliders like the LHC are constructed for exploring new physics at higher energies, they also provide a tremendous set of data for precise measurements within the Standard Model. The LHC will provide proton-proton collisions at energies of about $14 \mathrm{TeV}$ and at a rate of about $40 \mathrm{MHz}$. CMS is a general purpose detector which is designed to detect and measure the properties of the particles in the final state of these collisions. Detailed simulations of the expected events are created using Monte Carlo techniques. On the basis of these simulations and advanced data analysis techniques the potential for the precise Standard Model measurements can be studied. An overview is given of the results obtained within domains like top quark physics, electro-weak physics, gauge boson couplings, B-physics and QCD. Due to the high rate and energy it is expected that new Standard Model processes become visible at the LHC. The search for the Standard Model Higgs boson is described as the most important example.
\end{abstract}

International Europhysics Conference on High Energy Physics

July 21st - 27th 2005

Lisboa, Portugal

\footnotetext{
${ }^{*}$ Speaker.

'On behalf of the CMS Collaboration, speaker supported by FWO-Vlaanderen.
} 


\section{Design Parameters of the Experiment: LHC and CMS}

The Large Hadron Collider is designed to deliver proton collisions at a centre-of-mass energy of $14 \mathrm{TeV}$ with a rate of $40 \mathrm{MHz}$ reaching a luminosity of $10^{34} \mathrm{~cm}^{-2} \mathrm{~s}^{-1}$. The Compact Muon Solenoid will be positioned around one of the beam crossing points. This multi-purpose detector with a solenoid producing a 4 Tesla magnetic field along the beam line will perform efficient tracking up to pseudo-rapidities $|\eta|<2.4$, electro-magnetic calorimetry up to $|\eta|<3.0$, hadron calorimetry up to $|\eta|<5.2$ and muon detection up to $|\eta|<2.4$. Several physics results can be achieved with this experiment of which some are highlighted below. One year of data taking will correspond to $10 \mathrm{fb}^{-1}$.

\section{Top Quark Physics}

The determination of the properties of the top quark remains an essential part in testing the Standard Model. Compared to the Tevatron, where they were discovered, the LHC will be a top quark factory at about one $\mathrm{t} \overline{\mathrm{t}}$ pair per second with a NLO cross-section of $\sigma_{\overline{\mathrm{t}}} \simeq 800 \mathrm{pb}$. The singlelepton $t \bar{t}$ final state can be selected with a high $\mathrm{S} / \mathrm{N}$ ratio. Fitting the top quark mass spectrum in Figure 1 results in a statistical uncertainty of $0.25 \mathrm{GeV} / \mathrm{c}^{2}$, while the dominating systematic uncertainties could reach values of around $1 \mathrm{GeV} / \mathrm{c}^{2}$ and arize mainly from radiation effects and the calibration of the absolute jet energy. An alternative top mass estimator has been constructed from
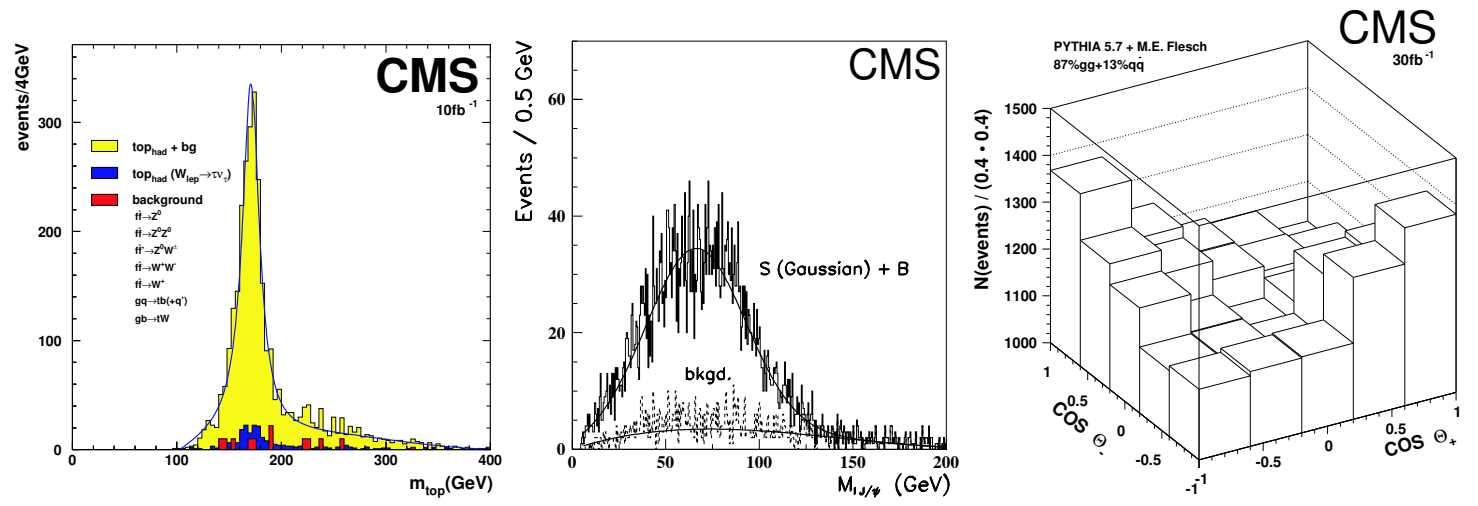

Figure 1: Left: reconstructed top quark mass distribution. Middle: distribution of the invariant mass of the lepton and the $\mathrm{J} / \psi$ system. Right: double differential distribution of the cosine of the lepton angles $\theta_{1}^{*}$.

these single-lepton events where a $\mathrm{J} / \psi \rightarrow \mu \mu$ meson is formed in the $\mathrm{b}$ quark decay. The mass distribution of the muonic decay of the $\mathrm{J} / \psi$ and the lepton from the $\mathrm{W}$ decay in the same top quark is shown in Figure 1. This mass can be related to a top quark mass estimator in the high luminosity regime of LHC with smaller and different systematic uncertainties (b quark fragmentation) compared to the direct top mass estimator. Because of its very short lifetime the top quark does not form bound states and its spin information is not diluted by hadron formation. Spin correlations are parametrized by the LR-asymmetry $\mathscr{A}$ and can be estimated by fitting the double differential distribution

$$
\frac{1}{N} \cdot \frac{d^{2} N}{d \cos \theta_{l^{+}}^{*} d \cos \theta_{l^{-}}^{*}}=\frac{1}{4}\left(1-\mathscr{A} \cos \theta_{l^{+}}^{*} \cos \theta_{l^{-}}^{*}\right)
$$


shown in Figure 1, where the angles $\theta_{1^{ \pm}}^{*}$ are between the lepton in the top quark rest frame and the top quark in the $\mathrm{t} \overline{\mathrm{t}}$ rest frame. With the $\mathrm{qq} / \mathrm{gg} \rightarrow \mathrm{t} \overline{\mathrm{t}}$ production mixture at the LHC the resulting assymmetry is expected to be $\mathscr{A}=0.311 \pm 0.035$ (stat) \pm 0.028 (syst). Also single top quark processes will be visible in difference production channels (s-, t- and Wt-channel) each sensitive to new physics phenomena (respectively heavy $\mathrm{W}^{\prime}, \mathrm{FCNC}$ and $\mathrm{H}^{ \pm}$). The cross-section is also related to $\left|\mathrm{V}_{\mathrm{tb}}\right|$ at the percent level.

\section{B-physics}

The LHC/CMS data will allow for a rich B-physics program due to its precise tracking and vertexing. A new tagging method to measure the angle $\beta$ in the unitarity triangle by the decay $\mathrm{B}_{\mathrm{d}}^{0} \rightarrow \mathrm{J} / \psi \mathrm{K}_{\mathrm{s}}^{0}$ has been studied. The nature of the $\mathrm{b}$ quark can be tagged by the charge of the emitted pion in the $B^{* *}$ decay: $\mathrm{B}^{* *+} \rightarrow \mathrm{B}_{\mathrm{d}}^{0} \pi^{+}$. The assymetry between $\mathrm{B}_{\mathrm{d}}^{0}$ and $\overline{\mathrm{B}}_{\mathrm{d}}^{0}$ is related to $\beta$. The expected precision for $\beta$ with this method has been evaluated to be $\delta(\sin 2 \beta)=0.022\left(10 \mathrm{fb}^{-1}\right)$. The reconstructed mass of the $\mathrm{B}^{0} \pi$ system after event selection is shown in Figure 2.
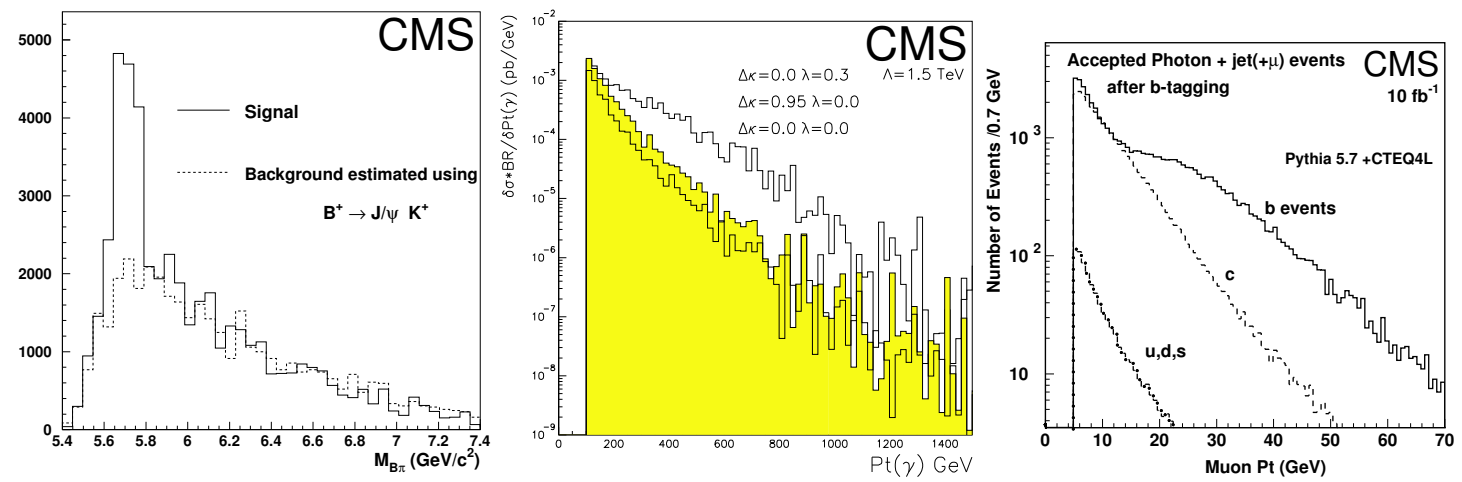

Figure 2: Left: reconstructed $\mathrm{B}^{0} \pi$ mass distribution. Middle: photon $\mathrm{p}_{\mathrm{T}}$ spectrum for several gauge boson couplings. Right: muon $\mathrm{p}_{\mathrm{T}}$ distribution of $\gamma+\mathrm{jet}(\mu)$ events.

\section{Electro-Weak Physics}

The Drell-Yan process $\mathrm{pp} \rightarrow \mathrm{1}^{+} \mathrm{l}^{-}$to be observed at the LHC up to much higher masses $\mathrm{M}_{\mathrm{1}^{+} \mathrm{1}^{-}}$ compared to the Tevatron is a measure for $\mathscr{A}_{F B}$ and hence $\sin ^{2} \theta_{\mathrm{eff}}^{\text {lept }}$. The precision on $\sigma_{1^{+} 1^{-}}$will exceed the magnitude of the EW corrections up to $\mathrm{M}_{1^{+} 1^{-}}=2 \mathrm{TeV} / \mathrm{c}^{2}$. Using forward lepton tagging the precision on $\sin ^{2} \theta_{\text {eff }}^{\text {lept }}$ could reach 0.00014 , with the main systematic uncertainty from the knowledge of the PDF's. Inversely the PDF's could be constraints by the measure of $\sin ^{2} \theta_{\text {eff }}^{\text {lept }}$.

\section{Gauge Boson Couplings}

Direct measurements of vector boson couplings are possible via the cross-section measurements of the processes in which they appear. They test the non-Abelian nature of the Standard Model gauge theory. Anomalous couplings or new physics can be included in the effective Lagrangian at a fundamental scale $\Lambda$. The $W \gamma$ coupling can be studied via the $\mathrm{p}_{\mathrm{T}}(\gamma)$ distribution shown in Figure 2. For $100 \mathrm{fb}^{-1}$ with $\Lambda=2 \mathrm{TeV}$, the expected 95\% CL limits are $|\Delta \kappa|<0.1$ and 
$|\lambda|<0.0009$. For $Z Z \gamma$ and $Z \gamma \gamma$ couplings both the $\mathrm{p}_{\mathrm{T}}(\gamma)$ and the $\mathrm{M}_{\mathrm{T}}(1 \mathrm{l} \gamma)$ spectra are sensitive to $\mathrm{h}_{\mathrm{i}}^{\mathrm{V}}(\mathrm{V}=\mathrm{Z}, \gamma)$ anomalous couplings.

\section{Parton Distribution Functions}

The knowledge of $\operatorname{PDF}\left(\mathrm{x}_{1}, \mathrm{x}_{2}, \mathrm{Q}^{2}\right)$ from HERA can be extrapolated via DGLAP evolutions to the LHC collisions. But also the huge LHC data itself can be used to determine the PDF's. The cross-section ratio of $W^{+}$and $W^{-}$production is related to $u(x) / d(x)$, while for the heavy quarks the associated production of $b / c$ (jet including a muon) with an isolated $\gamma$ or $\mathrm{W}$ with high $\mathrm{p}_{\mathrm{T}}$ can be used to determine their relative PDF's. The $\mathrm{p}_{\mathrm{T}}$ distribution of the muon in the $b / c$ jet is shown in Figure 2 and has clearly a different shape. In a similar way the gluon luminosity can be obtained with a $1 \%$ accuracy.

\section{Standard Model Higgs boson}

The SM-like scalar Higgs boson can be observed in several physics channels, depending on its mass $m_{H}$. In Figure 3 the sensitivity in the different channels is shown. The combined sensitive allows for a Higgs boson discovery in the range $114<m_{H}<800 \mathrm{GeV} / \mathrm{c}^{2}$ using $10 \mathrm{fb}^{-1}$.
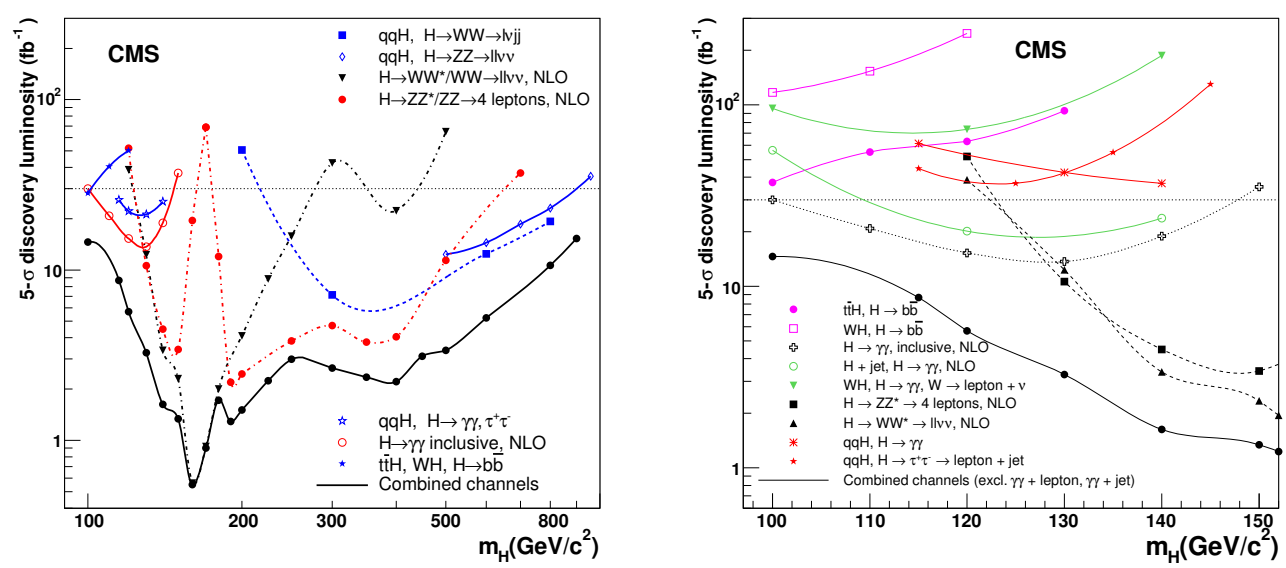

Figure 3: Integrated luminosity needed for discovery with statistical significance of $5 \sigma$ for the SM Higgs boson as a function of $\mathrm{m}_{\mathrm{H}}$.

\section{Summary}

It was shown with some key examples that the data accumulated by CMS at the LHC will allow to test the Standard Model with a significant improved precision compared to previous experiments. Also new particles (Higgs) or new processes (single-top) will become visible.

\section{References}

[1] CMS Collaboration physics results page:

http: //cmsdoc. cern. ch/cms/PRS/www/prs . php. 